\title{
Análisis del desempeño térmico de los sistemas constructivos de un edificio de oficinas mediante simulaciones dinámicas
}

\section{Thermal analysis of constructive systems at an office building through dynamic} simulations

\author{
Rodríguez-Muñoz Norma Alejandra \\ Consejo Nacional de Ciencia y Tecnología, México \\ Centro de Investigación en Materiales Avanzados, México \\ Departamento de Ingeniería Sustentable \\ Correo: norma.rodriguez@cimav.edu.mx \\ Nájera-Trejo Mario \\ Centro de Investigación en Materiales Avanzados, México \\ Departamento de Ingeniería Sustentable \\ Correo: mario.najera@cimav.edu.mx
}

\author{
Martín-Domínguez Ignacio Ramiro \\ Centro de Investigación en Materiales Avanzados, México \\ Departamento de Ingeniería Sustentable \\ Correo: ignacio.martin@cimav.edu.mx
}

\section{Resumen}

Se realizó el análisis térmico de un edificio de oficinas de tres plantas y $5500 \mathrm{~m}^{2}$ mediante el uso de un software de simulación dinámica. En el estudio se realizó un análisis del consumo energético anual por climatización artificial, variando los sistemas constructivos del edificio. El edificio estudiado se localiza en una región con clima BS1kw de acuerdo con la clasificación Köppen. Este clima, comúnmente referido como estepario, presenta condiciones en las cuales se requiere el uso de calefacción y refrigeración para proveer de confort térmico adecuado a través del año. Para este clima es posible incorporar diversas estrategias que contribuyan al ahorro energético. El análisis incorpora conceptos de arquitectura bioclimática y una selección de materiales de construcción y acristalado comúnmente utilizados. Para analizar el comportamiento térmico del edificio se implementaron diferentes sistemas de acristalado sobre el diseño arquitectónico propuesto, se estableció un rango de confort al interior del edificio, que a su vez funge como control de las temperaturas de operación del equipo de climatización. Los resultados muestran temperaturas promedio horarias y de consumo energético debido al acondicionamiento del aire de un año típico. Este trabajo se enfoca en argumentar que es posible disminuir el consumo energético de una edificación planeada o existente, mediante la evaluación de diversos sistemas constructivos convencionales comercialmente disponibles, sin modificar la geometría de la edificación.

Descriptores: Simulación dinámica, comportamiento térmico, edificaciones.

\begin{abstract}
A thermal analysis of an office building was performed with the use of a dynamic simulation software. This work focuses on the analysis of the constructive system contribution to the thermal performance of a $5500 \mathrm{~m}^{2}$ building. The studied building is located in a region within the BS1 kw climate according to the Köppen climate classification. In this climate commonly referred as steppe, both air cooling and heating is needed in order to provide a satisfactory thermal comfort condition throughout the year. Within this weather is possible to incorporate several energy saving strategies so the heat gains and losses can be reduced. The analysis starts with basic bioclimatic incorporations and the selection of common construction and glazing materials. In order to analyze the thermal behavior of the building, the implementation of different glazing materials at the designed building were proposed and the comfort temperature range was established as an operating parameter for the air conditioning units. The results show hourly mean temperatures and energetic demands due to air conditioning during the course of a year. This study is focused in discussing the possibility to reduce the energy requirement of a planned or existing building, by evaluating several conventional and commercially available constructive systems, without modifying the geometry of the building.
\end{abstract}

Keywords: Glazing, windows, dynamic simulation, thermal behavior. 


\section{INTRODUCCIÓN}

\section{USO DE ENERGÍA EN EDIFICACIONES}

En las últimas décadas la población mundial ha crecido de manera importante, alcanzándose la cifra de $7.3 \mathrm{mil}$ millones de habitantes en el 2015 (United Nations, 2015). Este aumento ha provocado entre otros efectos, el aumento de la demanda de toda clase de recursos y contaminación derivada de su uso y explotación. Observamos que la demanda energética ha aumentado, por ejemplo, el consumo de China se ha duplicado (IEA, 2008) mientras que de manera global se predice que el consumo energético de países en vías de desarro1 lo aumentará 3\% anual entre 2004 y 2020 (FAO, 2008). Existen parámetros que correlacionan el aumento en la población mundial y la demanda de energía mundial, tal como el PIB (Producto Interno Bruto). J. Darmstadter sugiere que a través del tiempo la relación de consumo entre sociedades con aumento en el PIB y la demanda energética han prevalecido (Darmstadter, 2004). El Panel Intergubernamental del Cambio Climático (IPCC) publicó un artículo dirigido al papel que tienen los individuos sobre el cambio climático, declarando que las emisiones de efecto invernadero se incrementaron cerca de 70\% entre 1970 y 2004 (IPCC, 2007). La demanda energética de los edificios entre 1990 y 2005 ha crecido rápidamente, donde los países de la OCDE han incrementado en $23 \%$ y $54 \%$ el uso de energía primaria y electricidad, respectivamente (IEA, 2008). La eficiencia energética juega un rol crítico en limitar el crecimiento de la demanda energética mundial, restricción que llega hasta 30\% para el 2040. Sin embargo, esto se logrará únicamente estableciéndose metas y regulaciones enérgicas alrededor del mundo.

El trabajar en el diseño de edificaciones sustentables, la ejecución de medidas de ahorro energético y la aplicación de conceptos bioclimáticos son totalmente pertinentes. La edificación sustentable utiliza prácticas y materiales respetuosos con el medio ambiente en todas sus fases: planeación, diseño, construcción, operación, etcétera (Álvarez, 2013). La eficiencia del uso de recursos en una edificación, contempla la reducción de demanda energética en la fase de operación, teniendo que las localidades donde se requiere el uso de climatización artificial, calefacción y refrigeración, son las que presentan mayor potencial de ahorro.

La tendencia actual es que el arquitecto diseña los espacios y el ingeniero define las instalaciones requeridas. La arquitectura bioclimática no solo debe basarse en el diseño arquitectónico y selección de materiales de construcción para alcanzar el confort térmico, sino debe incluir que la demanda energética requerida para enfriar y calentar la edificación sea la mínima posible (Givoni, 1994). En Latinoamérica, el diseño bioclimático es frecuentemente subestimado como una práctica válida dentro de las firmas arquitectónicas, donde los estudios de eficiencia energética o de edificación sustentable generalmente se basan solo en la identificación, aplicación de conceptos y adición de estrategias, es decir, aplicación de medidas cualitativas, sin considerar que es necesario calcular el desempeño térmico y energético de cada uno de los elementos añadidos. Es por esto que es necesario establecer una metodología que permita seleccionar las estrategias que consideren la arquitectura bioclimática, pero evaluando su impacto. Esta evaluación es posible que se efectue previo a la construcción de la edificación mediante simulación dinámica, e idealmente tanto para cuantificar la eficacia técnica de la estrategia como la económica, y así conjuntar las ventajas de la aplicación de la arquitectura bioclimática con la utilidad de cuantificar de forma ingenieril su implementación.

\section{ESTRATEGIAS DE AHORRO ENERGÉtICO EN EDIFICACIONES}

La localización, orientación, distribución de espacios, así como el aprovechamiento de las propiedades térmicas y ópticas de los materiales de construcción son características que contribuirán a conformar la demanda energética del edificio (Motawa y Oladokun, 2015). Bektas et al. (2011) dividen los parámetros que alteran los requerimientos energéticos del edificio como los parámetros medioambientales y los de diseño, clasificados como físicos y artificiales, respectivamente. Dentro de los parámetros físicos el autor resalta la importancia de la temperatura horaria exterior, la radiación solar y la velocidad y dirección del viento; mientras que los artificiales se refieren a la forma del edificio, proporción de acristalado, orientación, propiedades de materiales y la distancia entre los edificios.

Aksoy e Inalli (2006) estudiaron el impacto de estrategias pasivas en la demanda del sistema de calefacción, mediante un método de diferencias finitas. Las estrategias examinadas fueron la orientación y factor de forma, encontrando que el mejor factor de forma es $1 / 1$, es decir, edificios de forma cuadrada. Sadineni et al. (2011) realizan una revisión de componentes pasivos de la envolvente, analizando el estado del arte y propiedades de muros, ventanas y puertas, (acristalado y marcos) y cubierta. Señala que los elementos pasivos de una fachada son altamente sensibles a factores meteorológicos, por lo que el diseñador y planificador de la edificación deberá tener un amplio conocimiento de los 
elementos climáticos. Adicionalmente, para llevar a cabo un proyecto exitosamente, es recomendable analizar exhaustivamente la factibilidad económica resultante de la aplicación de las estrategias seleccionadas.

\section{USO DE SOFTWARE PARA ANÁLISIS ENERGÉTICO \\ DE EDIFICACIONES}

Existen diversos estudios donde se utilizan herramientas de simulación para cuantificar la demanda energética y el confort térmico en el interior de edificios. Crawley et al. (2008), realizaron un resumen donde comparan las capacidades de las herramientas y software disponibles durante los últimos 50 años. Los autores mencionan que aunque estos programas se han desarrollado, mejorado y utilizado por la comunidad para el diseño de edificaciones, encontraron que al grupo dedicado a la simulación le falta tener un lenguaje claro y uniforme para describir las características ofrecidas. A su vez Li et al. (2014) cuestionan los alcances de las herramientas de simulación energética en edificaciones, al establecer dos hipótesis que examinan las diferencias entre la información medida y calculada con las herramientas de simulación. La primera hipótesis se refiere a los errores incurridos por asumir la ocupación de la edificación, mientras que la segunda establece que al utilizarse un modelo energético que fue diseñado para otra estrategia de conservación de energía, resultarán predicciones estadísticamente incorrectas.

Autores como Nazi et al. (2015)y Li et al. (2014), utilizan Design Builder para cuantificar energía anual de edificios de oficinas. Nazi et al. (2015) aplican metodologías para reducir la carga de refrigeración en el verano, alcanzando una reducción del consumo energético del edificio de $45.85 \%$, mediante el ajuste de la temperatura de operación de los sistemas de enfriamiento y la modificación de los sistemas de iluminación y acristalado. Moreci et al. (2016), realizaron simulaciones con TRNSYS para establecer correlaciones simples para determinar las cargas de calefacción de edificios no residenciales para siete países europeos. El estudio se realizó con el fin de proveer con información a gerentes o planificadores energéticos sobre la demanda de calefacción de un edificio ideal puesto en diferentes ubicaciones geográficas, tomando en cuenta la normatividad reciente para cada sitio. En publicaciones de Florides et al. (2002) y Gieseler et al. (2004), se analiza la viabilidad económica del establecimiento de estrategias de ahorro energético sobre el consumo de energía. Mediante simulación dinámica con TRNSYS. Sadineni et al. (2011) realizan cálculos similares utilizando el programa computacional Energy-10.
PropiedADES DE LOS SISTEMAS CONSTRUCTIVOS Y COMPONENTES

Desde hace décadas se visualizaba la importancia que tiene la elección del acristalado en una edificación, en donde algunos retos que se concebían eran: el mejoramiento de calidad de la iluminación y aislamiento al ruido, la prevención de la fluctuación de la temperatura en las superficies interiores y la reducción de pérdidas y ganancias de calor a través del cristal, entre otros. Actualmente existe gran variedad de tipos de cristales, con propiedades ópticas muy diferentes, que permiten seleccionar los más adecuados al tipo de aplicación requerida. El conocimiento de las propiedades térmicas y ópticas será clave para el entendimiento y selección del tipo de cristal adecuado para cierta edificación (Orlov et al., 1998).

Baetens et al. (2010) concluyen de una revisión de literatura que las ventanas electrocrómicas son las más prometedoras comercialmente, ya que pueden reducir hasta $26 \%$ el consumo de energía requerida para iluminación, y hasta $20 \%$ el consumo energético por refrigeración cuando se utilizan en climas cálidos. A reserva de realizar más investigaciones para climas templados. Sweitzer et al. (1987) estudiaron el efecto de cristales de baja emisividad $($ low- $\varepsilon$ ) en edificios no residenciales, concluyendo que es posible ahorrar energía para iluminación y refrigeración en climas cálidos y templados, cuidando que las propiedades de transmisividad solar sean las adecuadas. En climas templados los recubrimientos de baja emisividad contribuyen a reducir los costos de calefacción, disminuyendo la condensación e incrementándose el confort térmico.

\section{Cálculo de demanda energética}

Li et al. (2014) consideran los consumos de calefacción, refrigeración, iluminación y equipo de cómputo y de oficina, demostrando que este edificio de oficinas se encuentra dentro de una clasificación de bajo consumo energético, consumiendo 1099123.74 kWh al año, lo que representa un índice de consumo de energía de $99.94 \mathrm{kWh} / \mathrm{m}^{2}$. En un estudio previo de los mismos autores se analizó el impacto de la selección de materiales de construcción sobre el consumo energético de otro edificio, dentro del mismo complejo. Como resultado se logró reducir la demanda de refrigeración anual hasta $37 \%$, mediante la selección de los materiales y la implementación de una estrategia de ventilación.

Ihara et al. (2015) se enfocaron en componentes de la envolvente que afectan la demanda energética. Se calculó la demanda energética de calefacción y refrigeración con la herramienta de simulación WUFI Plus. Las varia- 
ciones de fachada que estudiaron los autores son la reflectividad solar y valor $U$ de materiales de elementos opacos y SHGC (Solar Heat Gain Coefficient) de ventanas. Los autores encontraron que la reducción de SHGC de las ventanas fue la estrategia que impactó mayormente en la reducción de la demanda energética anual, seguido de la reducción del valor $\mathrm{U}$ de las ventanas.

Hee et al. (2015), analizan a detalle los efectos sobre el ahorro energético obtenido por la selección de diferentes tipos de acristalado, tomando en cuenta los efectos contradictorios entre el ahorro energético obtenido en climatización, y del mayor gasto por iluminación artificial derivada de la selección de los cristales. Dentro de su publicación incluyen una sección de lecciones aprendidas donde señalan que aunque la variedad de cristales disponibles en el mercado es amplia y tecnológicamente avanzada, el entendimiento de la aplicación de ciertos cristales puede traer conclusiones contradictorias, por lo que recomienda un proceso de optimización para su aplicación. $\mathrm{Y}$ al igual que Sadineni et al. (2011), hacen énfasis en el requerimiento de un buen conocimiento del clima de la región, ya que el desempeño de los cristales es altamente dependiente de el.

En este estudio se llevó a cabo un análisis del comportamiento térmico de una edificación, tomándose en cuenta las condiciones climáticas del lugar, geometría de la edificación, propiedades de los materiales, rango de confort térmico, horario de ocupación y condiciones de operación de los sistemas de climatización. Se realizó el análisis del edificio asumiendo la utilización de materiales comunes disponibles en la localidad. La finalidad de este trabajo es demostrar que es posible mejorar el desempeño térmico y disminuir el consumo energético de una edificación llevando a cabo su estudio, sin cambiar el diseño arquitectónico propuesto y sin proponer la utilización de materiales nuevos. En este trabajo se implementaron una serie de combinaciones de materiales y sistemas constructivos, para posteriormente cuantificar la mejora o deterioro de la eficiencia energética de la envolvente, considerando que una estrategia útil en tiempo de invierno puede afectar el comportamiento térmico de la edificación. Esta estrategia se siguió sin demeritar la importancia de la adecuación del proyecto arquitectónico y el uso de materiales novedosos, sino como un primer paso para la mejora del comportamiento de la edificación.

\section{Condiciones De OPERACIÓN DEL SISTEMA Y METODOlOGíA}

Este estudio se enfoca en analizar la contribución que tienen los sistemas constructivos sobre el comportamiento térmico de un edificio de tres plantas a través del software de simulación dinámica TRNSYS 17. El edificio comprende tres plantas: sótano, planta baja y planta alta, se dividió en zonas térmicas las cuales facilitan la evaluación y análisis (Figura 1).
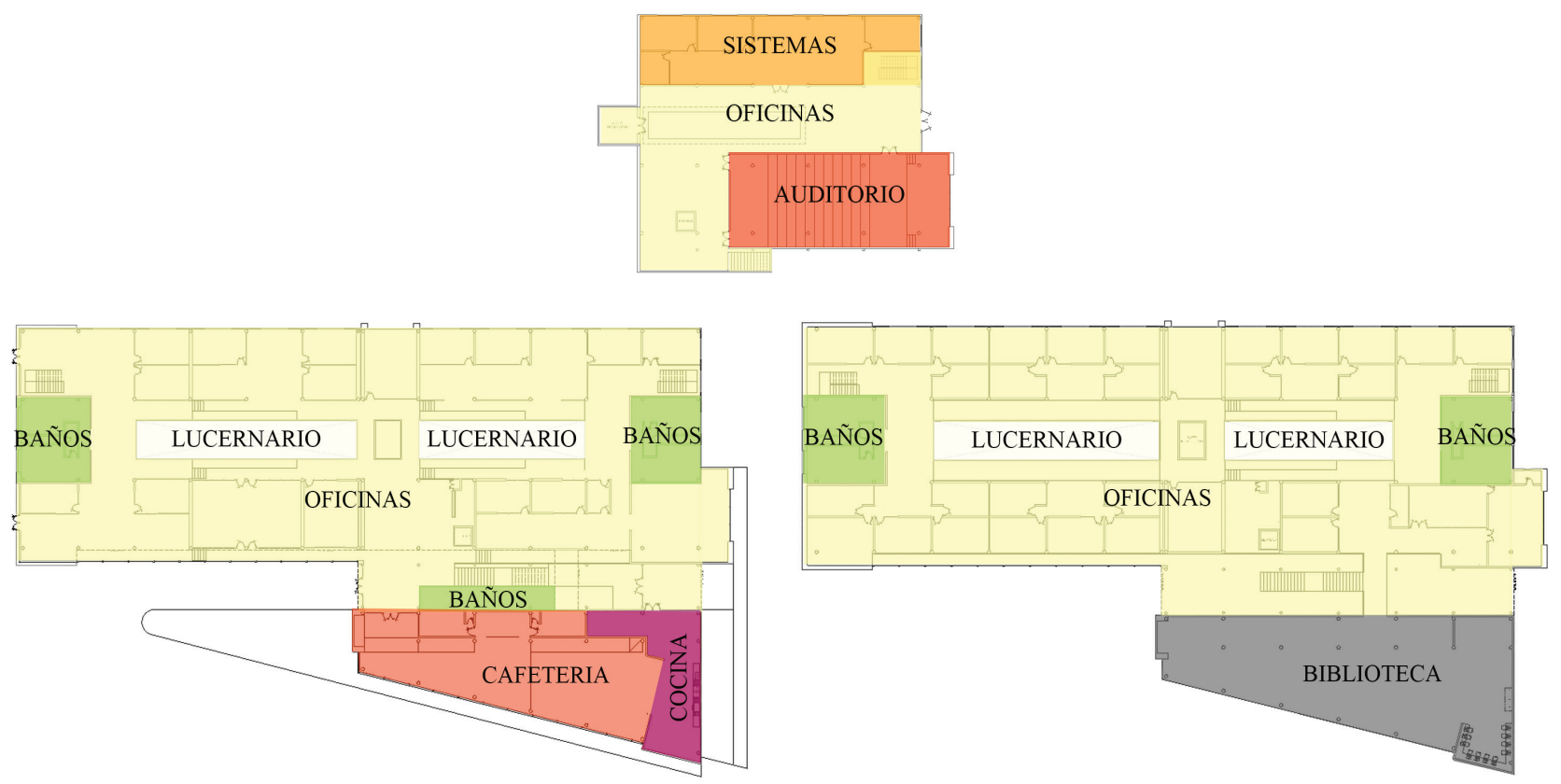

Figura 1. Zonas térmicas definidas: a) sótano, b) primera planta y c) segunda planta 
El análisis bioclimático y el estudio del desempeño térmico de la edificación, requiere datos climáticos del sitio de estudio a lo largo de todo el año para modelar la interacción clima y envolvente de la edificación. Para realizar el análisis de un año específico se trabajó con los datos de ese año. Pero, si se busca una estimación a largo plazo; se usa un año representativo de las condiciones climáticas promedio (Sengupta et al., 2015). Un dato popular obtenido por modelación es el TMY (Año meteorológico típico) de una ubicación específica.

El conjunto de datos que integra el TMY se diseñó originalmente para simplificar el cálculo de la climatización de las edificaciones. El TMY tipifica las condiciones meteorológicas a un sitio de interés y está basado en varios años de datos meteorológicos. Crawley et al. (2008) describen que el método TMY produce años sintéticos que representan las variables meteorológicas de manera más apropiada y harán predicciones de resultados más cercanos a los promedios a largo plazo. Remund (2015) habla sobre su fácil uso y una baja variación de la incertidumbre de $2-10 \%$. Autores como Hong et al. (2013) y Crawley et al. (2008) coinciden con que Meteonorm y el TMY son las herramientas más adecuadas a la hora de pensar en datos de entrada para la simulación de energía de edificaciones por ser la representación más cercana a los patrones típicos de clima.

Del reporte del IPCC (Intergovernmental Panel on Climate Change), se incluyeron el promedio de 18 modelos de predicción climática y tres escenarios de cambio climático en Meteonorm. Estos tres escenarios, B1, A1B y A2, están disponibles para predicciones futuras. Las anomalías de temperatura, precipitación, radiación global para los periodos 2011-2030, 2046-2065, 20802099 se usaron para el cálculo de los periodos de tiempo futuros. De acuerdo con estos cálculos, se encontró que la predicción de los cambios en la radiación global al 2100 en todos los escenarios son pequeños comparados con los cambios de temperatura. Estas variaciones se encuentran en el rango desde un décimo hasta un punto porcentual. Sin embargo, es importante resaltar que las variaciones de los últimos 50 años se han subestimado por los modelos anteriormente utilizados.

Los parámetros climáticos que se tomaron en cuenta en este estudio son: temperaturas horarias promedio, humedad, radiación solar y velocidad del viento, las cuales se introdujeron en forma de TMY (Typical Meteorological Year) a través del software Meteonorm. Por tratarse de un edificio de oficinas, se establecieron los días de ocupación de lunes a viernes en un horario de 07:00 a 19:00 horas, por lo que el resto del tiempo la climatización permanece apagada, como sucede de igual forma para los fines de semana. El establecimien- to de la ocupación de la edificación limita el uso de los sistemas de acondicionamiento de aire a estos periodos. Se estableció un rango de operación de los sistemas de climatización de 20 a $28^{\circ} \mathrm{C}$, donde se cuantifica la energía necesaria para calentar o enfriar cada zona térmica al momento de incumplirse con este parámetro.

Un sistema constructivo es un conjunto de materiales característicos para un tipo de construcción. Actualmente el sistema constructivo tradicional se refiere al proceso constructivo cuyo objetivo es la realización de muros de ladrillo, piedra, bloques u hormigón armado. En este trabajo se implementaron los sistemas constructivos y corridas de simulación para conocer el comportamiento térmico y energético del edificio, las cuales se detallan en la Tabla 1, en donde se observa que se evaluará el efecto que tiene la selección de impermeabilizante sobre la cubierta, del sistema constructivo de muros y de los cristales de las ventanas. Las propiedades térmicas y ópticas de los diferentes impermeabilizantes, muros y cristales utilizados se detallan en las Tablas 2, 3 y 4, respectivamente.

El caso inicial se simuló sin considerar sistemas de climatización artificial, obteniéndose el confort y temperaturas promedio del interior, representándose únicamente la protección brindada por la envolvente bajo las condiciones climáticas de un año típico. Las corridas de simulación posteriores se realizan con el fin de cuantificar la demanda energética del edificio debida al uso de sistemas de acondicionamiento cuando las condiciones de confort establecidas no se cumplen, variando la configuración de cubierta, muros y acristalado.

La geometría del edificio se creó utilizando el software Google SketchUp 8 y la información se extrajo utilizando planos de AutoCAD. Además de la geometría, las dimensiones de la cristalería, la orientación, el sombreado y las zonas térmicas del edificio son características que se definieron en SketchUp mediante el modulo añadido de TRNSYS. En TRNBuild se realiza la importación del modelo del edificio para posteriormente modificar las propiedades físicas de los materiales tales como, espesor, densidad, capacidad calorífica, conductividad térmica, coeficiente de absortancia y emisividad de la superficie. Este módulo de TRNSYS cuenta con la posibilidad de realizar un balance de energía basado en el control de temperatura del nodo de aire en la zona térmica. El rango utilizado para el control de temperatura se establece como $20^{\circ} \mathrm{C}$ para la calefacción y $28^{\circ} \mathrm{C}$ para la refrigeración. De esta forma, el módulo cuantifica de manera simplificada el consumo energético por climatización artificial. Usando TRNSYS Simulation Studio se incorpora la información climatológica y se manipula la información de salida de la edificación. 
Tabla 1. Establecimiento de los casos de estudio

\begin{tabular}{|c|c|c|c|c|}
\hline & Clave & Muros & Cristal & Cubierta \\
\hline \multirow{2}{*}{$\begin{array}{c}\text { Evaluación } \\
\text { impermeabilizantes }\end{array}$} & IMP-01 & \multirow{2}{*}{ Block concreto } & \multirow{2}{*}{$\begin{array}{l}\text { Cristal claro } \\
\text { convencional }\end{array}$} & $\begin{array}{l}\text { Impermeabilizante } \\
\text { Terracota }\end{array}$ \\
\hline & IMP-02 & & & $\begin{array}{c}\text { Impermeabilizante Blanco } \\
\text { reflectivo }\end{array}$ \\
\hline \multirow{3}{*}{$\begin{array}{l}\text { Evaluación uso de } \\
\text { materiales de construcción }\end{array}$} & MURO-01 & Block concreto & $\begin{array}{l}\text { Cristal claro } \\
\text { convencional }\end{array}$ & \multirow{8}{*}{$\begin{array}{l}\text { Impermeabilizante } \\
\text { Terracota }\end{array}$} \\
\hline & MURO-02 & Hebel & $\begin{array}{l}\text { Cristal claro } \\
\text { convencional }\end{array}$ & \\
\hline & MURO-03 & $\begin{array}{c}\text { Block concreto } \\
+0.0254 \mathrm{~m} \text { de } \\
\text { poliestireno }\end{array}$ & $\begin{array}{l}\text { Cristal claro } \\
\text { convencional }\end{array}$ & \\
\hline \multirow{5}{*}{$\begin{array}{l}\text { Evaluación tipo de } \\
\text { acristalado en ventanas }\end{array}$} & CRISTAL-01 & Block concreto & $\begin{array}{l}\text { Cristal claro } \\
\text { convencional }\end{array}$ & \\
\hline & CRISTAL-02 & Block concreto & Cristal claro & \\
\hline & CRISTAL-03 & Block concreto & Cristal control solar & \\
\hline & CRISTAL-04 & Block concreto & $\begin{array}{l}\text { Cristal baja } \\
\text { emisividad } 1\end{array}$ & \\
\hline & CRISTAL-05 & Block concreto & $\begin{array}{c}\text { Cristal baja } \\
\text { emisividad } 2\end{array}$ & \\
\hline
\end{tabular}

Tabla 2. Propiedades térmicas y ópticas de los materiales en cubierta

\begin{tabular}{|c|c|c|c|c|c|c|}
\hline Clave & $\begin{array}{l}\text { Composición de } \\
\text { cubierta }\end{array}$ & $\begin{array}{l}\text { Espesor } \\
(\mathrm{m})\end{array}$ & $\begin{array}{c}\text { Conductividad térmica } \\
\mathrm{k}(\mathrm{W} / \mathrm{m} \mathrm{K})\end{array}$ & $\begin{array}{c}\text { Emisividad } \\
(\varepsilon)\end{array}$ & $\begin{array}{c}\text { Absortividad } \\
(\alpha)\end{array}$ & $\begin{array}{c}\text { Reflectividad } \\
(\rho)\end{array}$ \\
\hline \multirow{3}{*}{ IMP-01 } & I. Terracota & 0.0254 & 0.100 & \multirow{3}{*}{0.35} & \multirow{3}{*}{0.60} & \multirow{3}{*}{0.40} \\
\hline & Concreto & 0.1000 & 1.400 & & & \\
\hline & Metal deck & 0.0100 & 45.28 & & & \\
\hline \multirow{3}{*}{ IMP-02 } & I. Blanco reflectivo & 0.0254 & 0.063 & \multirow{3}{*}{0.85} & \multirow{3}{*}{0.30} & \multirow{3}{*}{0.70} \\
\hline & Concreto & 0.1000 & 1.400 & & & \\
\hline & Metal deck & 0.0100 & 45.28 & & & \\
\hline
\end{tabular}

Tabla 3. Propiedades térmicas de los materiales en muros

\begin{tabular}{|c|c|c|c|c|}
\hline CLAVE & \multicolumn{2}{|c|}{ Configuración muro } & Espesor (m) & $\begin{array}{c}\text { Conductividad térmica } \\
\mathrm{k}(\mathrm{W} / \mathrm{m} \mathrm{K})\end{array}$ \\
\hline \multirow{2}{*}{ MURO-01 } & exterior & $\begin{array}{l}\text { Block de } \\
\text { concreto }\end{array}$ & 0.2000 & 1.63 \\
\hline & interior & Yeso & 0.0130 & 0.16 \\
\hline \multirow{3}{*}{ MURO-02 } & exterior & Poliestireno & 0.0254 & 0.17 \\
\hline & centro & $\begin{array}{l}\text { Block de } \\
\text { concreto }\end{array}$ & 0.2000 & 1.63 \\
\hline & interior & Yeso & 0.0130 & 0.16 \\
\hline \multirow{2}{*}{ MURO-03 } & exterior & Hebel & 0.1500 & 0.48 \\
\hline & interior & Yeso & 0.0130 & 0.16 \\
\hline
\end{tabular}


Tabla 4. Propiedades de cristales

\begin{tabular}{|c|c|c|c|c|c|}
\hline \multirow{2}{*}{ CLAVE } & \multirow{2}{*}{$\begin{array}{l}\text { Espesor } \\
(\mathrm{m})\end{array}$} & \multirow{2}{*}{$\begin{array}{c}\text { Coeficiente de ganancia } \\
\text { solar } \\
\text { SHGC }\end{array}$} & \multirow{2}{*}{$\begin{array}{l}\text { Factor U } \\
\left(\mathrm{W} / \mathrm{m}^{2} \mathrm{~K}\right)\end{array}$} & \multicolumn{2}{|c|}{$\begin{array}{c}\text { Emisividad } \\
(\varepsilon)\end{array}$} \\
\hline & & & & Interior & Exterior \\
\hline CRISTAL-01 & 0.016 & 0.855 & 5.68 & 0.84 & 0.84 \\
\hline CRISTAL-02 & 0.016 & 0.696 & 5.14 & 0.84 & 0.84 \\
\hline CRISTAL-03 & 0.016 & 0.495 & 5.42 & 0.84 & 0.84 \\
\hline CRISTAL-04 & 0.016 & 0.472 & 5.40 & 0.67 & 0.84 \\
\hline CRISTAL-05 & 0.016 & 0.612 & 2.04 & 0.84 & 0.88 \\
\hline
\end{tabular}

\section{DisCUSIÓN Y ANÁLISIS DE RESULTADOS}

Con el fin de demostrar que el consumo energético de una edificación se puede reducir al realizar la selección de materiales, aun con disponibilidad limitada de ellos, se realizó el análisis detallado del comportamiento térmico a través de simulaciones dinámicas, presentándose el requerimiento energético de los sistemas de calefacción y refrigeración de un edificio de tres plantas y 5500 $\mathrm{m}^{2}$ de superficie. De acuerdo con la evaluación del comportamiento de temperaturas dentro del edificio base mostrado en la Figura 2, se decidió evaluar los siguientes componentes constructivos: cubierta (impermeabilizante), muros y acristalado. En la Figura 2, se grafican las temperaturas interior $\left(\mathrm{T}_{\mathrm{i}}\right)$ y ambiental $\left(\mathrm{T}_{\mathrm{a}}\right)$, obteniéndose $\mathrm{T}_{\mathrm{i}}$ promedio, mínima y máxima anuales de $22.5,4.5$ y $33.4^{\circ} \mathrm{C}$, respectivamente, mientras que las $\mathrm{T}_{\mathrm{a}}$ promedio, mínima y máxima anuales fueron de $18.5,-3.8$ y $35.3^{\circ} \mathrm{C}$. En el año típico analizado se encontró que durante un total de 2070 horas al año se tendrán condiciones fuera del rango de confort establecido, por lo que se requerirá del uso de los sistemas de climatización.
En las Figuras 3, 4 y 5 se realiza una comparación del consumo de energía anual para los diferentes sistemas estudiados (la nomenclatura de los sistemas evaluados puede encontrarse en la Tabla 1).

Para el análisis de cubierta (Figura 3), se seleccionaron dos impermeabilizantes: color terracota y color blanco con propiedades reflectivas. Además se consideraron dos condiciones de sombreado en los lucernarios, debido a que estos representan pérdidas y ganancias de calor significativas. En la Figura 3, las claves (a) y (b) en el análisis de cubierta, se refieren a lucernarios sin sombreado y con sombreado, respectivamente. En la comparación de energía requerida los casos IMP-b (con sombreado) son efectivos para la reducción del requerimiento de energía para refrigerar, reduciéndose hasta un 31 y $44 \%$ la demanda cuando se compara contra los casos IMP-a (sin sombreado). Sin embargo, la medida resulta contraproducente al examinar los consumos por calefacción, donde la misma medida que ahorra $44 \%$ de energía, requiere $26 \%$ más energía que el caso base (IMP-01-a: sin sombreado e impermeabilizante terracota). Al realizar la suma anual de los consumos, se obser-

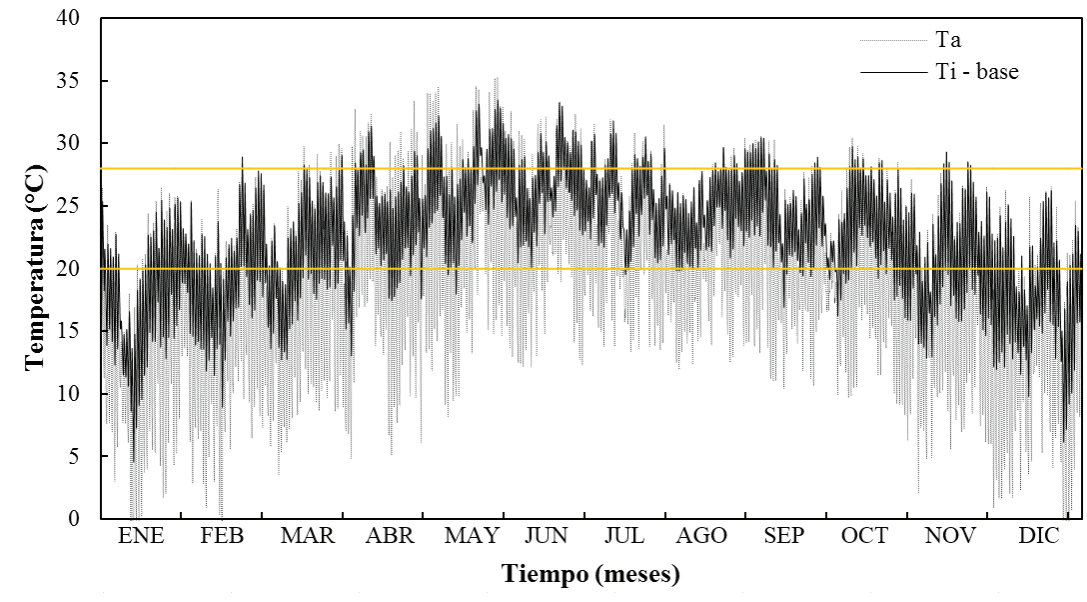

Figura 2. Temperaturas interiores promedio alcanzadas en el edificio base sin climatizar 
va que para esta configuración de edificio y clima la estrategia que funciona mejor es IMP-01-b: con sombreado e impermeabilizante terracota), el cual disminuirá hasta $4.63 \%$ el consumo de energía del edificio.

Para el análisis de muros (Figura 4), el sistema constructivo que resultaría en un menor consumo energético es el MURO-02: (bloque de concreto y poliestireno). Este sistema reduce $18.8 \%$ el consumo energético anual. Este sistema resulta efectivo especialmente para la reducción de la demanda energética por calefacción, con una reducción de $23 \%$ de la demanda prevista. Esta mejora se debe principalmente a la inclusión del material aislante (poliestireno, $\mathrm{k}=0.17 \mathrm{~W} / \mathrm{m} \mathrm{K}$ ) en el sistema constructivo. Por lo que este sistema reduciría de mane- ra importante las pérdidas de calor a través de la envolvente del edificio en los meses fríos.

Por último, se analizaron cinco sistemas de acristalado (Figura 5), donde todos resultan favorables para la reducción del flujo de calor al interior del edificio. Sin embargo, al cuantificar el consumo de energía correspondiente a calefacción se observa que todos los sistemas analizados consumen más energía respecto al sistema de acristalado convencional, esto principalmente a los valores bajos del coeficiente de ganancia de calor solar (SHGC) que poseen los demás cristales. Un alto coeficiente SHGC, nos indica que el cristal permitirá mayores ganancias de calor, mientras que un coeficiente bajo, permitirá poco flujo de calor. En el caso del sistema
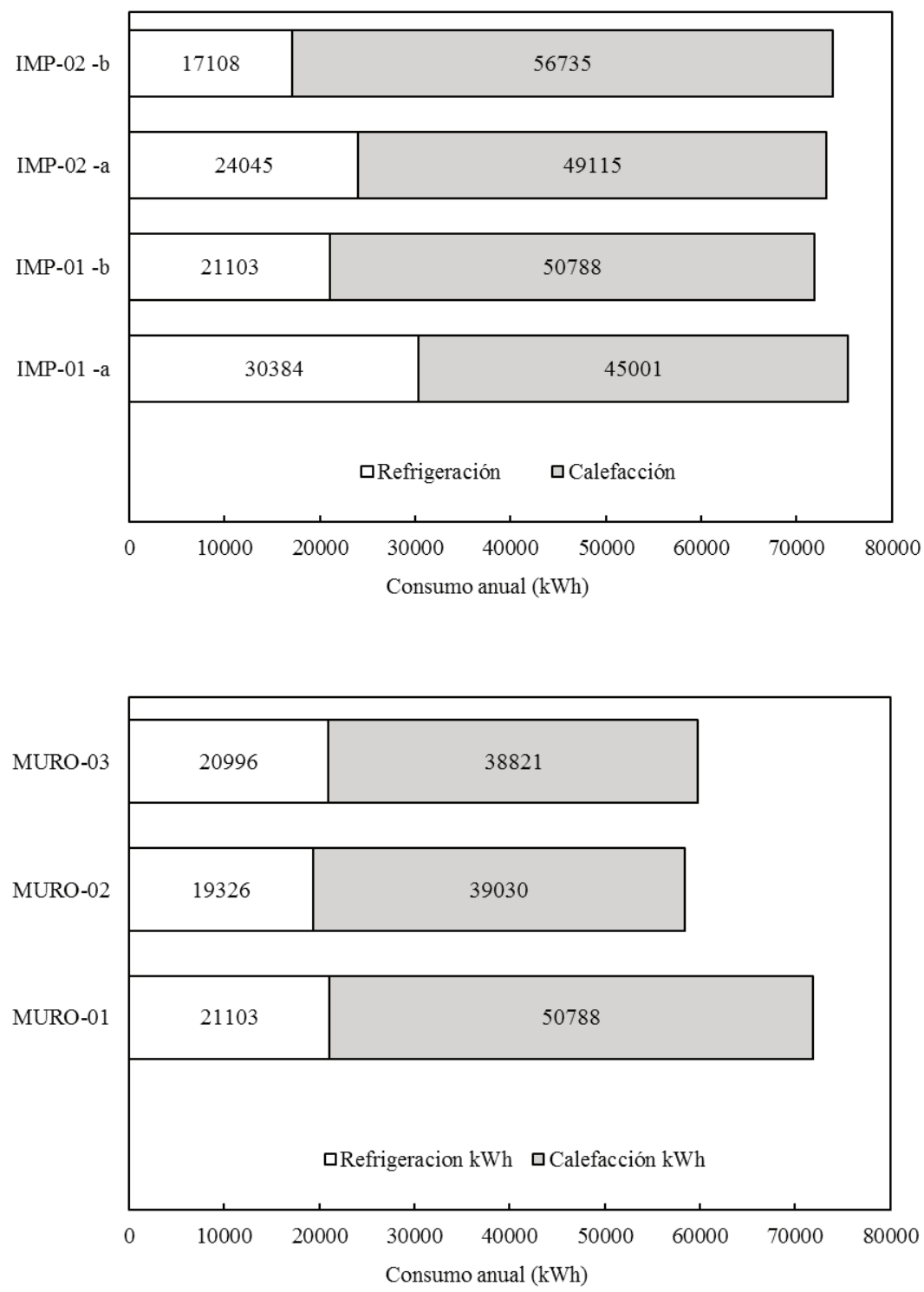

Figura 3. Comparativo de consumo energético anual en cubierta
Figura 4. Comparativo de consumo energético anual en muro 


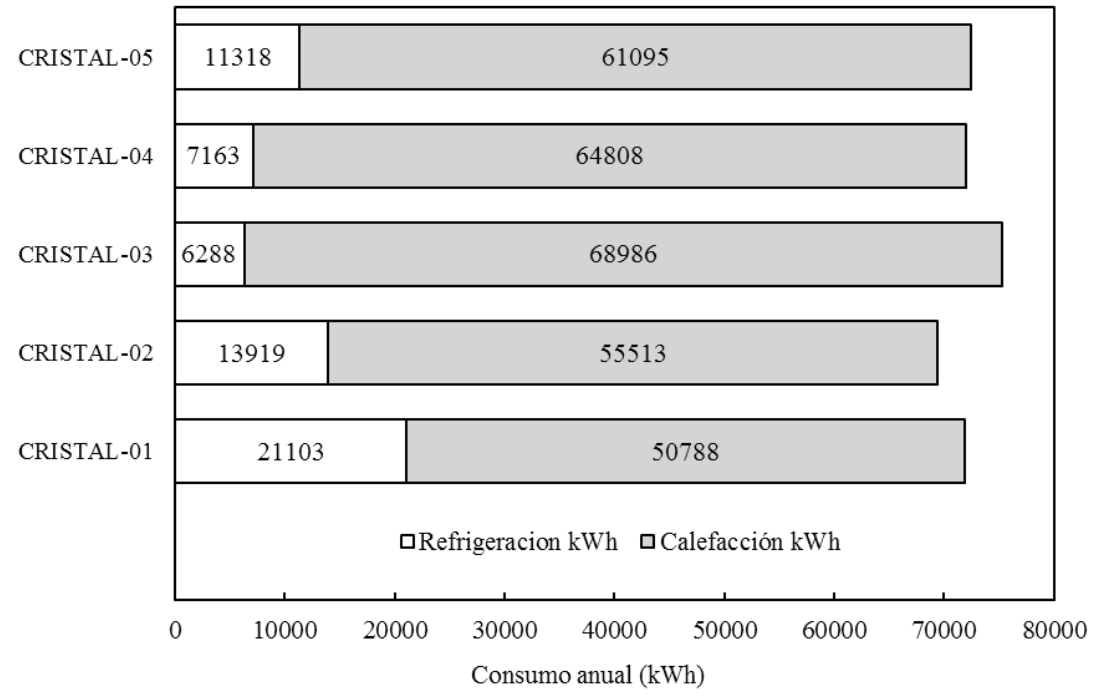

Figura 5. Comparativo de consumo energético anual en ventana convencional, este tiene un coeficiente de 0.855 , indicando mayores ganancias de calor, lo cual se traducirá en una demanda de calefacción menor. El sistema CRISTAL-03, tiene un SHGC menor (0.472), observándose que es el sistema que tendría mayor demanda de calefacción (68986 kWh). Cuando se realiza un balance de consumo anual de ambos requerimientos (refrigeración y calefacción), el sistema CRISTAL-02 (Cristal claro) logra reducir en $3.4 \%$ la demanda energética anual respecto al caso base, CRISTAL-01(Cristal convencional).

\section{CONCLuSiones}

En este estudio se examinó el desempeño térmico de sistemas constructivos propuestos para un edificio de oficinas en clima BS mediante simulación dinámica con el software TRNSYS. Se propusieron dos sistemas constructivos para cubierta, tres para muro y tres de acristalado para un edificio de tres plantas $\left(5500 \mathrm{~m}^{2}\right)$.

Uno de los objetivos de este trabajo fue visualizar que es posible disminuir el consumo energético de una edificación, aún con disponibilidad limitada de materiales de construcción. Con los resultados derivados del estudio se demostró que no es necesario realizar un estudio complejo para reducir el consumo de energía por concepto de refrigeración y calefacción manteniendo el confort en su interior.

De acuerdo con el análisis efectuado, se encontró que de los tres elementos variados (impermeabilizante, muro y cristal) el elemento que tiene mayor impacto sobre la disminución del consumo energético es el aislamiento de los muros, mientras que el elemento que en segundo término mejora el desempeño térmico fue la aplicación de un impermeabilizante color terracota. Por último, la consideración de los diferentes cristales en las ventanas tiene un menor impacto en este edificio. Esto da una pauta inicial para decidir qué elementos constructivos tienen mayor impacto en el comportamiento térmico de una edificación, principalmente cuando es necesario priorizar la selección de materiales debido a restricciones presupuestales.

Finalmente, al analizar el consumo energético anual del edificio base contra el del edificio propuesto, los resultados demuestran que el consumo energético por refrigeración se logra reducir en mayor cantidad que por calefacción. No obstante, la reducción en el gasto operativo anual respecto a la edificación propuesta es de hasta $25.5 \%$, lográndose así el objetivo principal de este análisis.

Es importante hacer énfasis en que el análisis de una edificación debiera realizarse siempre de forma anual, ya que frecuentemente se analiza la incorporación de una o varias estrategias enfocadas a mejorar las condiciones de verano o invierno, sin tomar en cuenta que dichas estrategias ayudan a mejorar el desempeño térmico en invierno pero afectan el de verano y viceversa, lo cual trae como consecuencia la neutralización de las ganancias o ahorros prometidos por la incorporación de alguna de las estrategias.

\section{AgradeCimientos}

Se agradece el apoyo económico recibido por parte del: Centro Mexicano de Innovación en Energía Solar (CeMIE-Sol), A través del Proyecto: P13 “Laboratorios de pruebas para baja y media temperatura, laboratorio para 
el diseño e integración de sistemas termo solares asistido por computadora" Perteneciente a la Convocatoria 201302, del: FONDO SECTORIAL CONACYT - SENER SUSTENTABILIDAD ENERGÉTICA. Para el desarrollo y presentación de este trabajo. Asimismo al proyecto FOMIX-Durango: DGO-2014-01-229107: “Creación del Centro de Innovación y Competitividad en Energías Renovables y Medio Ambiente de Durango".

\section{RefERENCIAS}

Aksoy U.T. e Inalli M. Impacts of some building passive design parameters on heating demand for a cold region. Building and Environment, volumen 41 (número 12), 2006: 1742-1754. ISSN 03601323. DOI 10.1016/j.buildenv.2005.07.011.

Álvarez-García G. Energía en Edificaciones. Revista Mexicana de Física, volumen 59 (número 2013) 2013: 44-51.

Baetens R., Jelle B.P., Gustavsen A. Properties, requirements and possibilities of smart windows for dynamic daylight and solar energy control in buildings: A state-of-the-art review. Solar Energy Materials and Solar Cells [en línea], volumen 94 (número 2), 2010: 87-105. ISSN 09270248. DOI 10.1016/j.solmat.2009.08.021. Disponible en: http://dx.doi.org/10.1016/j. solmat.2009.08.021.

Bektas-Ekici B. y Aksoy.U.T. Prediction of building energy needs in early stage of design by using ANFIS. Expert Systems with Applications [en línea], volumen 38 (número 5), 2011: 53525358. ISSN 09574174. DOI 10.1016/j.eswa.2010.10.021. Disponible en: http://dx.doi.org/10.1016/j.eswa.2010.10.021.

Crawley D.B., Hand J.W., Kummert M., Griffith B.T. Contrasting the capabilities of building energy performance simulation programs. Building and Environment, volumen 43 (número 4), 2008: 661-673.

Darmstadter J. Energy and Population, (número septiembre), 2004: 1-9.

FAO, 2008. Forests and energy [en línea]. S.1.: s.n. ISBN 9789251 059852. Disponible en: ftp://ftp.fao.org/docrep/fao/010/i0139s/ i0139s00.pdf.

Florides G., Tassou S., Kalogirou S., Wrobel L. Measures used to lower building energy consumption and their cost effectiveness. Applied Energy [en línea], volumen 73 (números 3-4), 2002: 299-328. ISSN 03062619. DOI 10.1016/S0306-2619(02) 00119-8. Disponible en: http://www.sciencedirect.com/science/article/pii/S0306261902001198.

Gieseler U.D.J., Heidt F.D., Bier W. Evaluation of the cost efficiency of an energy efficient building. Renewable Energy, volumen 29 (número 3), 2004: 369-376. ISSN 09601481. DOI 10.1016/ S0960-1481(03)00254-4.

Givoni B. Passive Low Energy Cooling of Buildings. 1a ed., S.1.,Wiley, 1994. ISBN 978-0-471-28473-4.

Hee W.J., Alghoul M.A., Bakhtyar B., Elayeb O., Shameri M.A., Alrubaih M.S., Sopian, K. The role of window glazing on da- ylighting and energy saving in buildings. Renewable and Sustainable Energy Reviews [en línea], volumen 42, 2015: 323-343. ISSN 1364-0321. DOI 10.1016/j.rser.2014.09.020. Disponible en: http://dx.doi.org/10.1016/j.rser.2014.09.020.

Hong T., Chang W.K., Lin H.W. A fresh look at weather impact on peak electricity demand and energy use of buildings using 30-year actual weather data. Applied Energy [en línea], volumen 111, 2013: 333-350. ISSN 03062619. DOI 10.1016/j.apenergy.2013.05.019. Disponible en: http://dx.doi.org/10.1016/j. ape\%20nergy.2013.05.019.

IEA, 2008. Worldwide Trends in Energy Use and Efficiency. Iea. Org [en línea], pp. 93. Disponible en: http://iea.org/efficiency/ CD-EnergyEfficiencyPolicy2009/1-Croos-sectoral/1-Indicators_2008.pdf.

Ihara T., Gustavsen A., Jelle B.P. Effect of facade components on energy efficiency in office buildings. Applied Energy [en línea], volumen 158, 2015: 422-432. ISSN 03062619. DOI 10.1016/j. apenergy.2015.08.074. Disponible en: http://dx.doi. org/10.1016/\%20j.apenergy.2015.08.074.

IPCC, 2007. Climate Change 2007 Synthesis Report. S.1.: s.n. ISBN 9291691224.

Li H., Wang W., Feng G., Ding H., Zheng X. Proceedings of the 8th International Symposium on Heating, Ventilation and Air Conditioning. Lecture Notes in Electrical Engineering [en línea]. S.1.: s.n., 2014: 101-108. ISBN 978-3-642-39580-2. DOI 10.10 07/978-3-642-39581-9. Disponible en: http://www.scopus.com/ inward/record.url?eid=2-s2.0-84894175153\&partnerID=tZOtx 3y1.

Meehl G.A., Stocker T.F., Collins W.D., Friedlingstein P., Gaye A.T., Gregory J.M., Kitoh A., Knutti R., Murphy J.M., Noda A., Raper S.C.B., Watterson I.G., Weaver A.J., Zhao Z.-C. 2007: Global Climate Projections. Climate Change 2007: Contribution of Working Group I to the Fourth Assessment Report of the Intergovernmental Panel on Climate Change [en línea]. S.1.: Disponible en: http://www.ipcc.ch/pdf/assessment-report/ar4/wg1/ar4wg1-chapter10.pdf.

Moreci E., Ciulla G., Lo-Brano V. Annual heating energy requirements of office buildings in a European climate. Sustainable Cities and Society [en línea], volumen 20, 2016: 81-95. ISSN 22106707. DOI 10.1016/j.scs.2015.10.005. Disponible en: http:// dx.doi.org/10.1016/j.scs.2015.10.005.

Motawa I. y Oladokun M. A model for the complexity of household energy consumption. Energy and Buildings [en línea], volumen 87, 2015: 313-323. ISSN 03787788. DOI 10.1016/j. enbuild.2014.11.044. Disponibleen: http://dx.doi.org/10.1016/j. enbuild.2014.11.044.

Nazi W.I.W., Wang Y.D., Roskilly T. Methodologies to Reduce Cooling Load using Heat Balance Analysis: A Case Study in an Office Building in a Tropical Country. Energy Procedia, volumen 75 (número 0), 2015: 1269-1274. ISSN 18766102. DOI 10.1016/j.egypro.2015.07.185. 
Orlov D.L., Chesnokov A.G., Gorln A.E., Emel O.A., Problems of Energy Efficient Glazing, volumen 55, 1998: 3-7. ISSN 03617610.

Remund J. Accuracy of Meteonorm [en línea]. 2015. S.1.: s.n. Disponible en: www.meteotest.ch.

Sadineni S.B., France T.M., Boehm R.F. Economic feasibility of energy efficiency measures in residential buildings. Renewable Energy, volumen 36 (número 11), 2011:. 2925-2931. ISSN 09601481. DOI 10.1016/j.renene.2011.04.006.

Sadineni S.B., Madala S., Boehm R.F. Passive building energy savings: A review of building envelope components. Renewable and Sustainable Energy Reviews [en línea], volumen 15 (número 8), 2011:. 3617-3631. ISSN 13640321. DOI 10.1016/j.rser.2011. 07.014. Disponible en: http://dx.doi.org/10.1016/j. rser.2011.\%2007.014

Sengupta M., Habte A., Kurtz S., Dobo, A., Wilbert S., Lorenz E., Stoffel T., Renné D., Gueymard C., Myers D., Wilcox S., Blanc P., Perez R. Best Practices Handbook for the Collection and Use of Solar Resource Data for Solar Energy Applications Best Practices Handbook for the Collection and Use of Solar Resource Data for Solar Energy Applications, 2015,. S.1.: s.n. ISBN 0038-092X.

Sweitzer G., Arasteh D., Selkowitz S. Effects of Low-Emissivity Glazings on Energy Use Patterns in Non-Residential Daylighted Buildings. 1987. S.1.: s.n.
United Nations. World Population Prospects. World Population Prospects: The 2015 Revision, Key Findings and Advance Tables. S.1.: 2015.

\section{Este artículo se cita:}

\section{Citación estilo Chicago}

Rodríguez-Muñoz, Norma Alejandra, Mario Nájera-Trejo, Ignacio Ramiro Martín-Domínguez. Análisis del desempeño térmico de los sistemas constructivos de un edificio de oficinas mediante simulaciones dinámicas. Ingeniería Investigación y Tecnología, XIX, 03 (2018): 279289.

\section{Citación estilo ISO 690}

Rodríguez-Muñoz N.A., Nájera-Trejo M., Martín-Domínguez I.R. Análisis del desempeño térmico de los sistemas constructivos de un edificio de oficinas mediante simulaciones dinámicas. Ingeniería Investigación y Tecnología, volumen XIX (número 3), julio-septiembre 2018: 279-289.

\section{SemblanZAS de LOS Autores}

Rodríguez-Muñoz Norma Alejandra. Ingeniera Química, con Maestría y Doctorado en Ciencias de la Ingeniería de la Universidad de Sonora. Fue posdoctorante dentro del proyecto de Laboratorio Nacional de Sistemas de Concenetración Solar y Química Solar (LACyQS) del Instituto de Energías Renovables de la UNAM. Actualmente es investigador de Cátedras CONACYT en el Departamento de Ingeniería Sustentable del Centro de Investigación en Materiales Avanzados en Durango. Nájera-Trejo Mario. Técnico Titular B en CIMAV-Unidad Durango. Es Ingeniero Electromecánico con especialidad en manufactura avanzada. Cuenta con Maestría en Ciencia y Tecnología Ambiental y participó en el análisis térmico de los edificios en construcción de la Unidad Durango. Participa en el proyecto CEMIESOL P13: Creación del laboratorio para diseño termo-solar asistido por computadora. Asimismo, en el proyecto de evaluación del desempeño de recubrimientos reflejantes/emisivos para techos de viviendas de la empresa COMEX.

Martín-Domínguez Ignacio Ramiro. Investigador en CIMAV-Unidad Durango. Ingeniero Mecánico por el Instituto Tecnológico de Durango, Doctorado en Termociencias por la Universidad de Windsor, Canadá. Sus líneas de investigación son: Modelación, simulación y diseño de sistemas termo mecánicos, aplicaciones de la energía solar térmica, docencia en temas de termofluidos, transferencia de calor, energía solar y simulación computacional de sistemas de energía. “ 
